\title{
DIAGONAL AND LOW-RANK MATRIX DECOMPOSITIONS, CORRELATION MATRICES, AND ELLIPSOID FITTING*
}

\author{
J. SAUNDERSON ${ }^{\dagger}$, V. CHANDRASEKARAN ${ }^{\ddagger}$, P. A. PARRILO ${ }^{\dagger}$, AND A. S. WILLSKY ${ }^{\dagger}$
}

\begin{abstract}
In this paper we establish links between, and new results for, three problems that are not usually considered together. The first is a matrix decomposition problem that arises in areas such as statistical modeling and signal processing: given a matrix $X$ formed as the sum of an unknown diagonal matrix and an unknown low-rank positive semidefinite matrix, decompose $X$ into these constituents. The second problem we consider is to determine the facial structure of the set of correlation matrices, a convex set also known as the elliptope. This convex body, and particularly its facial structure, plays a role in applications from combinatorial optimization to mathematical finance. The third problem is a basic geometric question: given points $v_{1}, v_{2}, \ldots, v_{n} \in \mathbb{R}^{k}$ (where $n>k$ ) determine whether there is a centered ellipsoid passing exactly through all the points. We show that in a precise sense these three problems are equivalent. Furthermore we establish a simple sufficient condition on a subspace $\mathcal{U}$ that ensures any positive semidefinite matrix $L$ with column space $\mathcal{U}$ can be recovered from $D+L$ for any diagonal matrix $D$ using a convex optimization-based heuristic known as minimum trace factor analysis. This result leads to a new understanding of the structure of rank-deficient correlation matrices and a simple condition on a set of points that ensures there is a centered ellipsoid passing through them.
\end{abstract}

Key words. elliptope, minimum trace factor analysis, Frisch scheme, semidefinite programming, subspace coherence

AMS subject classifications. 90C22, 52A20, 62H25, 93B30

DOI. $10.1137 / 120872516$

1. Introduction. Decomposing a matrix as a sum of matrices with simple structure is a fundamental operation with numerous applications. A matrix decomposition may provide computational benefits, such as allowing the efficient solution of the associated linear system in the square case. Furthermore, if the matrix arises from measurements of a physical process (such as a sample covariance matrix), decomposing that matrix can provide valuable insight about the structure of the physical process.

Among the most basic and well-studied additive matrix decompositions is the decomposition of a matrix as the sum of a diagonal matrix and a low-rank matrix. This decomposition problem arises in the factor analysis model in statistics, which has been studied extensively since Spearman's original work of 1904 [29]. The same decomposition problem is known as the Frisch scheme in the system identification literature [17]. For concreteness, in section 1.1 we briefly discuss a stylized version of a problem in signal processing that under various assumptions can be modeled as a (block-) diagonal and low-rank decomposition problem.

${ }^{*}$ Received by the editors April 5, 2012; accepted for publication (in revised form) by M. L. Overton October 17, 2012; published electronically December 19, 2012. This research was funded in part by Shell International Exploration and Production, Inc., under P.O. 450004440 and in part by the Air Force Office of Scientific Research under grant FA9550-11-1-0305.

http://www.siam.org/journals/simax/33-4/87251.html

$\dagger$ Laboratory for Information and Decision Systems, Department of Electrical Engineering and Computer Science, Massachusetts Institute of Technology, Cambridge, MA 02139 (jamess@mit.edu, parrilo@mit.edu, willsky@mit.edu). A preliminary version of parts of this work appeared in the master's thesis of the first author, Subspace Identification via Convex Optimization.

$¥$ Department of Computing and Mathematical Sciences, California Institute of Technology, Pasadena, CA 91125 (venkatc@caltech.edu). This work was completed while this author was with the Laboratory for Information and Decision Systems, Massachusetts Institute of Technology.

1395 
Much of the literature on diagonal and low-rank matrix decompositions is in one of two veins. An early approach [1] that has seen recent renewed interest [11] is an algebraic one, where the principal aim is to give a characterization of the vanishing ideal of the set of symmetric $n \times n$ matrices that decompose as the sum of a diagonal matrix and a rank $k$ matrix. Such a characterization has only been obtained for the border cases $k=1, k=n-1$ (due to Kalman [17]) and the recently resolved $k=2$ case (due to Brouwer and Draisma [3] following a conjecture by Drton, Sturmfels, and Sullivan [11]). This approach does not (yet) offer scalable algorithms for performing decompositions, rendering it unsuitable for many applications, including those in high-dimensional statistics, optics [12], and signal processing [24]. The other main approach to factor analysis is via heuristic local optimization techniques, often based on the expectation maximization algorithm [9]. This approach, while computationally tractable, typically offers no provable performance guarantees.

A third way is offered by convex optimization-based methods for diagonal and low-rank decompositions such as minimum trace factor analysis (MTFA), the idea and initial analysis of which dates at least to Ledermann's 1940 work [21]. MTFA is computationally tractable, being based on a semidefinite program (see section 2), and yet offers the possibility of provable performance guarantees. In this paper we provide a new analysis of MTFA that is particularly suitable for high-dimensional problems.

Semidefinite programming duality theory provides a link between this matrix decomposition heuristic and the facial structure of the set of correlation matricespositive semidefinite matrices with unit diagonal - also known as the elliptope [19]. This set is one of the simplest of spectrahedra - affine sections of the positive semidefinite cone. Spectrahedra are of particular interest for two reasons. First, spectrahedra are a rich class of convex sets that have many nice properties (such as being facially exposed). Second, there are well-developed algorithms, efficient both in theory and in practice, for optimizing linear functionals over spectrahedra. These optimization problems are known as semidefinite programs [30].

The elliptope arises in semidefinite programming-based relaxations of problems in areas such as combinatorial optimization (e.g., the MAX-CUT problem [14]) and statistical mechanics (e.g., the $k$-vector spin glass problem [2]). In addition, the problem of projecting onto the set of (possibly low-rank) correlation matrices has enjoyed considerable interest in mathematical finance and numerical analysis in recent years [16]. In each of these applications the structure of the set of low-rank correlation matrices, i.e., the facial structure of this convex body, plays an important role.

Understanding the faces of the elliptope turns out to be related to the following ellipsoid fitting problem: given $n$ points in $\mathbb{R}^{k}$ (with $n>k$ ), under what conditions on the points is there an ellipsoid centered at the origin that passes exactly through these points? While there is considerable literature on many ellipsoid-related problems, we are not aware of any previous systematic investigation of this particular problem.

1.1. Illustrative application: Direction of arrival estimation. Direction of arrival estimation is a classical problem in signal processing where (block-) diagonal and low-rank decomposition problems arise naturally. In this section we briefly discuss some stylized models of the direction of arrival estimation problem that can be reduced to matrix decomposition problems of the type considered in this paper.

Suppose we have $n$ sensors at locations $\left(x_{1}, y_{1}\right),\left(x_{2}, y_{2}\right), \ldots,\left(x_{n}, y_{n}\right) \in \mathbb{R}^{2}$ that are passively "listening" for waves (electromagnetic or acoustic) at a known frequency from $r \ll n$ sources in the far field (so that the waves are approximately plane waves when they reach the sensors). The aim is to estimate the number of sources $r$ and their 


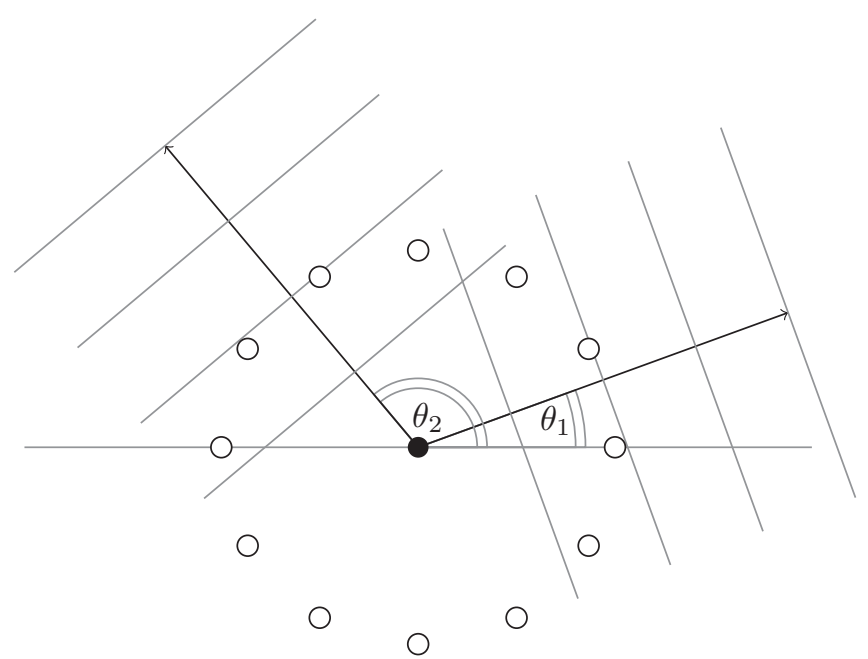

FIG. 1.1. Plane waves from directions $\theta_{1}$ and $\theta_{2}$ arriving at an array of sensors equally spaced on a circle (a uniform circular array).

directions of arrival $\theta=\left(\theta_{1}, \theta_{2}, \ldots, \theta_{r}\right)$ given sensor measurements and knowledge of the sensor locations (see Figure 1.1).

A standard mathematical model for this problem (see [18] for a derivation) is to model the vector of sensor measurements $z(t) \in \mathbb{C}^{n}$ at time $t$ as

$$
z(t)=A(\theta) s(t)+n(t),
$$

where $s(t) \in \mathbb{C}^{r}$ is the vector of baseband signal waveforms from the sources, $n(t) \in \mathbb{C}^{n}$ is the vector of sensor measurement noise, and $A(\theta)$ is the $n \times r$ matrix with complex entries $[A(\theta)]_{i j}=e^{-k \sqrt{-1}\left(x_{i} \cos \left(\theta_{j}\right)+y_{i} \sin \left(\theta_{j}\right)\right)}$ with $k$ a positive constant related to the frequency of the waves being sensed.

The column space of $A(\theta)$ contains all the information about the directions of arrival $\theta$. As such, subspace-based approaches to direction of arrival estimation aim to estimate the column space of $A(\theta)$ (from which a number of standard techniques can be employed to estimate $\theta$ ).

Typically $s(t)$ and $n(t)$ are modeled as zero-mean stationary white Gaussian processes with covariances $\mathbb{E}\left[s(t) s(t)^{H}\right]=P$ and $\mathbb{E}\left[n(t) n(t)^{H}\right]=Q$, respectively (where $A^{H}$ denotes the Hermitian transpose of $A$ and $\mathbb{E}[\cdot]$ the expectation). In the simplest setting, $s(t)$ and $n(t)$ are assumed to be uncorrelated so that the covariance of the sensor measurements at any time is

$$
\Sigma=A(\theta) P A(\theta)^{H}+Q .
$$

The first term is Hermitian positive semidefinite with rank $r$, i.e., the number of sources. Under the assumption that spatially well-separated sensors (such as in a sensor network) have uncorrelated measurement noise, $Q$ is diagonal. In this case the covariance $\Sigma$ of the sensor measurements decomposes as a sum of a positive semidefinite matrix of rank $r \ll n$ and a diagonal matrix. Given an approximation of $\Sigma$ (e.g., a sample covariance) approximately performing this diagonal and low-rank matrix decomposition allows the estimation of the column space of $A(\theta)$ and in turn the directions of arrival. 
A variation on this problem occurs if there are multiple sensors at each location, sensing, for example, waves at different frequencies. Again under the assumption that well-separated sensors have uncorrelated measurement noise, and sensors at the same location have correlated measurement noise, the sensor noise covariance matrix $Q$ would be block-diagonal. As such the covariance of all the sensor measurements would decompose as the sum of a low-rank matrix (with rank equal to the total number of sources over all measured frequencies) and a block-diagonal matrix.

A block-diagonal and low-rank decomposition problem also arises if the secondorder statistics of the noise have certain symmetries. This might occur in cases where the sensors themselves are arranged in a symmetric way (such as in the uniform circular array shown in Figure 1.1). In this case there is a unitary matrix $T$ (depending only on the symmetry group of the array) such that $T Q T^{H}$ is block-diagonal [25]. Then the covariance of the sensor measurements, when written in coordinates with respect to $T$, is

$$
T \Sigma T^{H}=T A(\theta) P A(\theta)^{H} T^{H}+T Q T^{H},
$$

which has a decomposition as the sum of a block-diagonal matrix and a rank $r$ Hermitian positive semidefinite matrix (as conjugation by $T$ does not change the rank of this term).

Note that the matrix decomposition problems discussed in this section involve Hermitian matrices with complex entries, rather than the symmetric matrices with real entries considered elsewhere in this paper. In Appendix B we briefly discuss how results for the complex case can be obtained from our results for the block-diagonal and low-rank decomposition problem over the reals.

\subsection{Contributions.}

Relating MTFA, correlation matrices, and ellipsoid fitting. We introduce and make explicit the links between the analysis of MTFA, the facial structure of the elliptope, and the ellipsoid fitting problem, showing that these problems are, in a precise sense, equivalent (see Proposition 3.1). As such, we relate a basic problem in statistical modeling (tractable diagonal and low-rank matrix decompositions), a basic problem in convex algebraic geometry (understanding the facial structure of perhaps the simplest of spectrahedra), and a basic geometric problem.

A sufficient condition for the three problems. The main result of the paper is to establish a new, simple, sufficient condition on a subspace $\mathcal{U}$ of $\mathbb{R}^{n}$ that ensures that MTFA correctly decomposes matrices of the form $D^{\star}+L^{\star}$, where $\mathcal{U}$ is the column space of $L^{\star}$. The condition is stated in terms of a measure of coherence of a subspace (made precise in Definition 4.1). Informally, the coherence of a subspace is a real number between zero and one that measures how close the subspace is to containing any of the elementary unit vectors. This result can be translated into new results for the other two problems under consideration based on the relationship between the analysis of MTFA, the faces of the elliptope, and ellipsoid fitting.

Block-diagonal and low-rank decompositions. In section 5 we turn our attention to the block-diagonal and low-rank decomposition problem, showing how our results generalize to that setting. Our arguments combine our results for the diagonal and low-rank decomposition case with an understanding of the symmetries of the blockdiagonal and low-rank decomposition problem.

1.3. Outline. The remainder of the paper is organized as follows. We describe notation, give some background on semidefinite programming, and provide precise 
problem statements in section 2 . In section 3 we present our first contribution by establishing relationships between the success of MTFA, the faces of the elliptope, and ellipsoid fitting. We then illustrate these connections by noting the equivalence of a known result about the faces of the elliptope, and a known result about MTFA, and translating these into the context of ellipsoid fitting. Section 4 is focused on establishing and interpreting our main result: a sufficient condition for the three problems based on a coherence inequality. Finally in section 5 we generalize our results to the analogous tractable block-diagonal and low-rank decomposition problem.

\section{Background and problem statements.}

2.1. Notation. If $x, y \in \mathbb{R}^{n}$ we denote by $\langle x, y\rangle=\sum_{i=1}^{n} x_{i} y_{i}$ the standard Euclidean inner product and by $\|x\|_{2}=\langle x, x\rangle^{1 / 2}$ the corresponding Euclidean norm. We write $x \geq 0$ and $x>0$ to indicate that $x$ is entrywise nonnegative and strictly positive, respectively. Correspondingly, if $X, Y \in \mathcal{S}^{n}$, the set of $n \times n$ symmetric matrices, then we denote by $\langle X, Y\rangle=\operatorname{tr}(X Y)$ the trace inner product and by $\|X\|_{F}=$ $\langle X, X\rangle^{1 / 2}$ the Frobenius norm. We write $X \succeq 0$ and $X \succ 0$ to indicate that $X$ is positive semidefinite and strictly positive definite, respectively. We write $\mathcal{S}_{+}^{n}$ for the cone of $n \times n$ positive semidefinite matrices.

The column space of a matrix $X$ is denoted $\mathcal{R}(X)$ and the nullspace is denoted $\mathcal{N}(X)$. If $X$ is an $n \times n$ matrix, then $\operatorname{diag}(X) \in \mathbb{R}^{n}$ is the diagonal of $X$. If $x \in \mathbb{R}^{n}$, then $\operatorname{diag}^{*}(x) \in \mathcal{S}^{n}$ is the diagonal matrix with $\left[\operatorname{diag}^{*}(x)\right]_{i i}=x_{i}$ for $i=1,2, \ldots, n$. If $\mathcal{U}$ is a subspace of $\mathbb{R}^{n}$, then $P_{\mathcal{U}}: \mathbb{R}^{n} \rightarrow \mathbb{R}^{n}$ denotes the orthogonal projector onto $\mathcal{U}$, that is, the self-adjoint linear map such that $\mathcal{R}\left(P_{\mathcal{U}}\right)=\mathcal{U}, P_{\mathcal{U}}^{2}=P_{\mathcal{U}}$ and $\operatorname{tr}\left(P_{\mathcal{U}}\right)=\operatorname{dim}(\mathcal{U})$.

We use the notation $e_{i}$ for the vector with a one in the $i$ th position and zeros elsewhere and the notation 1 to denote the vector all entries of which are one. We use the shorthand $[n]$ for the set $\{1,2, \ldots, n\}$. The set of $n \times n$ correlation matrices, i.e., positive semidefinite matrices with unit diagonal, is denoted $\mathcal{E}_{n}$. For brevity we typically refer to $\mathcal{E}_{n}$ as the elliptope and the elements of $\mathcal{E}_{n}$ as correlation matrices.

2.2. Semidefinite programming. The term semidefinite programming [30] refers to convex optimization problems of the form

$$
\underset{X}{\operatorname{minimize}}\langle C, X\rangle \text { subject to }\left\{\begin{array}{r}
\mathcal{A}(X)=b, \\
X \succeq 0,
\end{array}\right.
$$

where $X$ and $C$ are $n \times n$ symmetric matrices, $b \in \mathbb{R}^{m}$, and $\mathcal{A}: \mathcal{S}^{n} \rightarrow \mathbb{R}^{m}$ is a linear map. The dual semidefinite program is

$$
\underset{y, S}{\operatorname{maximize}}\langle b, y\rangle \text { subject to }\left\{\begin{aligned}
C-\mathcal{A}^{*}(y) & =S, \\
S & \succeq 0,
\end{aligned}\right.
$$

where $\mathcal{A}^{*}: \mathbb{R}^{m} \rightarrow \mathcal{S}^{n}$ is the adjoint of $\mathcal{A}$.

General semidefinite programs can be solved efficiently using interior point methods [30]. While our focus in this paper is not on algorithms, we remark that for the structured semidefinite programs discussed in this paper, many different specialpurpose methods have been devised.

The main result about semidefinite programming that we use is the following optimality condition (see [30], for example).

TheOREM 2.1. Suppose (2.1) and (2.2) are strictly feasible. Then $X^{\star}$ and $\left(y^{\star}, S^{\star}\right)$ are optimal for the primal (2.1) and dual (2.2), respectively, if and only if $X^{\star}$ is primal feasible, $\left(y^{\star}, S^{\star}\right)$ is dual feasible, and $X^{\star} S^{\star}=0$. 
2.3. Tractable diagonal and low-rank matrix decompositions. To decompose $X$ into a diagonal part and a positive semidefinite low-rank part, we may try to solve the following rank minimization problem:

$$
\underset{D, L}{\operatorname{minimize}} \operatorname{rank}(L) \quad \text { subject to }\left\{\begin{array}{l}
X=D+L \\
L \succeq 0 \\
D \text { diagonal. }
\end{array}\right.
$$

Since the rank function is nonconvex and discontinuous, it is not clear how to solve this optimization problem directly. One approach that has been successful for other rank minimization problems (for example, those in $[22,23]$ ) is to replace the rank function with the trace function in the objective. This can be viewed as a convexification of the problem as the trace function is the convex envelope of the rank function when restricted to positive semidefinite matrices with spectral norm at most one. Performing this convexification leads to the semidefinite program MTFA:

$$
\underset{D, L}{\operatorname{minimize}} \operatorname{tr}(L) \text { subject to }\left\{\begin{array}{c}
X=D+L, \\
L \succeq 0, \\
D \text { diagonal. }
\end{array}\right.
$$

It has been shown by Della Riccia and Shapiro [7] that if MTFA is feasible it has a unique optimal solution. One central concern of this paper is to understand when the diagonal and low-rank decomposition of a matrix given by MTFA is "correct" in the following sense.

Recovery problem $\mathrm{I}$. Suppose $X$ is a matrix of the form $X=D^{\star}+L^{\star}$, where $D^{\star}$ is diagonal and $L^{\star}$ is positive semidefinite. What conditions on $\left(D^{\star}, L^{\star}\right)$ ensure that $\left(D^{\star}, L^{\star}\right)$ is the optimum of MTFA with input $X$ ?

We establish in section 3 that whether $\left(D^{\star}, L^{\star}\right)$ is the optimum of MTFA with input $X=D^{\star}+L^{\star}$ depends only on the column space of $L^{\star}$, motivating the following definition.

Definition 2.2. A subspace $\mathcal{U}$ of $\mathbb{R}^{n}$ is recoverable by MTFA if for every diagonal $D^{\star}$ and every positive semidefinite $L^{\star}$ with column space $\mathcal{U},\left(D^{\star}, L^{\star}\right)$ is the optimum of MTFA with input $X=D^{\star}+L^{\star}$.

In these terms, we can restate the recovery problem succinctly as follows.

Recovery problem II. Determine which subspaces of $\mathbb{R}^{n}$ are recoverable by MTFA.

Much of the basic analysis of MTFA, including optimality conditions and relations between minimum rank factor analysis and minimum trace factor analysis, was carried out in a sequence of papers by Shapiro [26, 27, 28] and Della Riccia and Shapiro [7]. More recently, Chandrasekaran et al. [6] and Candès et al. [4] considered convex optimization methods for decomposing a matrix as a sum of a sparse and low-rank matrix. Since a diagonal matrix is certainly sparse, the analysis in [6] can be specialized to give fairly conservative sufficient conditions for the success of their convex programs in performing diagonal and low-rank decompositions (see section 4.1).

The diagonal and low-rank decomposition problem can also be interpreted as a low-rank matrix completion problem, where we are given all the entries of a low-rank matrix except the diagonal and aim to correctly reconstruct the diagonal entries. As such, this paper is closely related to the ideas and techniques used in the work of Candès and Recht [5] and a number of subsequent papers on this topic. We would like to emphasize a key point of distinction between that line of work and the present paper. The recent low-rank matrix completion literature largely focuses on determining the proportion of randomly selected entries of a low-rank matrix that need 
to be revealed to be able to reconstruct that low-rank matrix using a tractable algorithm. The results of this paper, on the other hand, can be interpreted as attempting to understand which low-rank matrices can be reconstructed from a fixed and quite canonical pattern of revealed entries.

2.4. Faces of the elliptope. The faces of the cone of $n \times n$ positive semidefinite matrices are all of the form

$$
\mathcal{F}_{\mathcal{U}}=\{X \succeq 0: \mathcal{N}(X) \supseteq \mathcal{U}\},
$$

where $\mathcal{U}$ is a subspace of $\mathbb{R}^{n}$ [19]. Conversely, given any subspace $\mathcal{U}$ of $\mathbb{R}^{n}, \mathcal{F}_{\mathcal{U}}$ is a face of $\mathcal{S}_{+}^{n}$. As a consequence, the faces of $\mathcal{E}_{n}$ are all of the form

$$
\mathcal{E}_{n} \cap \mathcal{F}_{\mathcal{U}}=\{X \succeq 0: \mathcal{N}(X) \supseteq \mathcal{U}, \operatorname{diag}(X)=\mathbf{1}\},
$$

where $\mathcal{U}$ is a subspace of $\mathbb{R}^{n}$ [19]. It is not the case, however, that for every subspace $\mathcal{U}$ of $\mathbb{R}^{n}$ there is a correlation matrix with nullspace containing $\mathcal{U}$, motivating the following definition.

Definition 2.3 (see [19]). A subspace $\mathcal{U}$ of $\mathbb{R}^{n}$ is realizable if there is an $n \times n$ correlation matrix $Q$ such that $\mathcal{N}(Q) \supseteq \mathcal{U}$.

The problem of understanding the facial structure of the set of correlation matrices can be restated as follows.

Facial structure problem. Determine which subspaces of $\mathbb{R}^{n}$ are realizable.

Much is already known about the faces of the elliptope. For example, all possible dimensions of faces as well as polyhedral faces are known [20]. Characterizations of the realizable subspaces of $\mathbb{R}^{n}$ of dimension $1, n-2$, and $n-1$ are given in [8] and implicitly in [19] and [20]. Nevertheless, little is known about which $k$-dimensional subspaces of $\mathbb{R}^{n}$ are realizable for general $n$ and $k$.

2.5. Ellipsoid fitting. Throughout, an ellipsoid is a set of the form $\left\{u \in \mathbb{R}^{k}\right.$ : $\left.u^{T} M u=1\right\}$, where $M \succeq 0$. Note that this includes "degenerate" ellipsoids.

Ellipsoid fitting problem I. What conditions on a collection of $n$ points in $\mathbb{R}^{k}$ ensure that there is a centered ellipsoid passing exactly through all those points?

Let us consider some basic properties of this problem.

Number of points. If $n \leq k$ we can always fit an ellipsoid to the points. Indeed if $V$ is the matrix with columns $v_{1}, v_{2}, \ldots, v_{n}$, then the image of the unit sphere in $\mathbb{R}^{n}$ under $V$ is a centered ellipsoid passing through $v_{1}, v_{2}, \ldots, v_{n}$. If $n>\left(\begin{array}{c}k+1 \\ 2\end{array}\right)$ and the points are "generic," then we cannot fit a centered ellipsoid to them. This is because if we represent the ellipsoid by a symmetric $k \times k$ matrix $M$, the condition that it passes through the points (ignoring the positivity condition on $M$ ) means that $M$ must satisfy $n$ linearly independent equations.

Invariances. If $T \in G L(k)$ is an invertible linear map, then there is an ellipsoid passing through $v_{1}, v_{2}, \ldots, v_{n}$ if and only if there is an ellipsoid passing through $T v_{1}, T v_{2}, \ldots, T v_{n}$. This means that whether there is an ellipsoid passing through $n$ points in $\mathbb{R}^{k}$ depends not on the actual set of $n$ points but on a subspace of $\mathbb{R}^{n}$ related to the points. We summarize this observation in the following lemma.

Lemma 2.4. Suppose $V$ is a $k \times n$ matrix with row space $\mathcal{V}$. If there is a centered ellipsoid in $\mathbb{R}^{k}$ passing through the columns of $V$, then there is a centered ellipsoid passing through the columns of any matrix $\tilde{V}$ with row space $\mathcal{V}$.

Lemma 2.4 asserts that whether it is possible to fit an ellipsoid to $v_{1}, v_{2}, \ldots, v_{n}$ depends only on the row space of the matrix with columns given by the $v_{i}$, motivating the following definition. 
Definition 2.5. A k-dimensional subspace $\mathcal{V}$ of $\mathbb{R}^{n}$ has the ellipsoid fitting property if there is a $k \times n$ matrix $V$ with row space $\mathcal{V}$ and a centered ellipsoid in $\mathbb{R}^{k}$ that passes through each column of $V$.

As such we can restate the ellipsoid fitting problem as follows.

Ellipsoid fitting problem II. Determine which subspaces of $\mathbb{R}^{n}$ have the ellipsoid fitting property.

3. Relating ellipsoid fitting, diagonal and low-rank decompositions, and correlation matrices. In this section we show that the ellipsoid fitting problem, the recovery problem, and the facial structure problem are equivalent in the following sense.

Proposition 3.1. Let $\mathcal{U}$ be a subspace of $\mathbb{R}^{n}$. Then the following are equivalent:

1. $\mathcal{U}$ is recoverable by $M T F A$.

2. $\mathcal{U}$ is realizable.

3. $\mathcal{U}^{\perp}$ has the ellipsoid fitting property.

Proof. Let $\operatorname{dim}(\mathcal{U})=n-k$. To see that 2 implies 3 , let $V$ be a $k \times n$ matrix with nullspace $\mathcal{U}$ and let $v_{i}$ denote the $i$ th column of $V$. If $\mathcal{U}$ is realizable there is a correlation matrix $Y$ with nullspace containing $\mathcal{U}$. Hence there is some $M \succeq 0$ such that $Y=V^{T} M V$ and $v_{i}^{T} M v_{i}=1$ for $i \in[n]$. Since $V$ has nullspace $\mathcal{U}$, it has row space $\mathcal{U}^{\perp}$. Hence the subspace $\mathcal{U}^{\perp}$ has the ellipsoid fitting property. By reversing the argument we see that the converse also holds.

The equivalence of 1 and 2 arises from semidefinite programming duality. Following a slight reformulation, MTFA (2.3) can be expressed as

$$
\underset{d, L}{\operatorname{maximize}}\langle\mathbf{1}, d\rangle \quad \text { subject to } \quad\left\{\begin{aligned}
X & =\operatorname{diag}^{*}(d)+L, \\
L & \succeq 0
\end{aligned}\right.
$$

and its dual as

$$
\underset{Y}{\operatorname{minimize}}\langle X, Y\rangle \text { subject to }\left\{\begin{aligned}
\operatorname{diag}(Y) & =\mathbf{1}, \\
Y & \succeq 0,
\end{aligned}\right.
$$

which is clearly just the optimization of the linear functional defined by $X$ over the elliptope. We note that (3.1) is exactly in the standard dual form (2.2) for semidefinite programming and correspondingly that (3.2) is in the standard primal form (2.1) for semidefinite programming.

Suppose $\mathcal{U}$ is recoverable by MTFA. Fix a diagonal matrix $D^{\star}$ and a positive semidefinite matrix $L^{\star}$ with column space $\mathcal{U}$ and let $X=D^{\star}+L^{\star}$. Since (3.1) and (3.2) are strictly feasible, by Theorem 2.1 (optimality conditions for semidefinite programming), the pair $\left(\operatorname{diag}\left(D^{\star}\right), L^{\star}\right)$ is an optimum of (3.1) if and only if there is some correlation matrix $Y^{\star}$ such that $Y^{\star} L^{\star}=0$. Since $\mathcal{R}\left(L^{\star}\right)=\mathcal{U}$ this implies that $\mathcal{U}$ is realizable. Conversely, if $\mathcal{U}$ is realizable, there is some $Y^{\star}$ such that $Y^{\star} L^{\star}=0$ for every $L^{\star}$ with column space $\mathcal{U}$, showing that $\mathcal{U}$ is recoverable by MTFA.

Remark. We note that in the proof of Proposition 3.1 we established that the two versions of the recovery problem stated in section 2.3 are actually equivalent. In particular, whether $\left(D^{\star}, L^{\star}\right)$ is the optimum of MTFA with input $X=D^{\star}+L^{\star}$ depends only on the column space of $L^{\star}$.

3.1. Certificates of failure. We can certify that a subspace $\mathcal{U}$ is realizable by constructing a correlation matrix with nullspace containing $\mathcal{U}$. We can also establish that a subspace is not realizable by constructing a matrix that certifies this fact. Geometrically, a subspace $\mathcal{U}$ is realizable if and only if the subspace $\mathcal{L}_{\mathcal{U}}=\left\{X \in \mathcal{S}^{n}\right.$ : 
$\mathcal{N}(X) \supseteq \mathcal{U}\}$ of symmetric matrices intersects with the elliptope. So a certificate that $\mathcal{U}$ is not realizable is a hyperplane in the space of symmetric matrices that strictly separates the elliptope from $\mathcal{L}_{\mathcal{U}}$. The following lemma describes the structure of these separating hyperplanes.

LEMma 3.2. A subspace $\mathcal{U}$ of $\mathbb{R}^{n}$ is not realizable if and only if there is a diagonal matrix $D$ such that $\operatorname{tr}(D)>0$ and $v^{T} D v \leq 0$ for all $v \in \mathcal{U}^{\perp}$.

Proof. By Proposition 3.1, $\mathcal{U}$ is not realizable if and only if $\mathcal{U}^{\perp}$ does not have the ellipsoid fitting property. Let $\operatorname{dim}\left(\mathcal{U}^{\perp}\right)=k$ and let $V$ be a $k \times n$ matrix with row space $\mathcal{U}^{\perp}$. Then $\mathcal{U}^{\perp}$ does not have the ellipsoid fitting property if and only if we cannot find an ellipsoid passing through the columns of $V$, i.e., the semidefinite program

$$
\underset{M}{\operatorname{minimize}}\langle 0, M\rangle \quad \text { subject to }\left\{\begin{aligned}
\operatorname{diag}\left(V^{T} M V\right) & =\mathbf{1}, \\
M & \succeq 0,
\end{aligned}\right.
$$

is infeasible. The semidefinite programming dual of (3.3) is

$$
\underset{d}{\operatorname{maximize}}\langle d, \mathbf{1}\rangle \quad \text { subject to } \quad\left\{V \operatorname{diag}^{*}(d) V^{T} \preceq 0 .\right.
$$

Both the primal and dual problems are strictly feasible, so strong duality holds. Since (3.4) is clearly always feasible, (3.3) is infeasible if and only if (3.4) is unbounded (by strong duality). This occurs if and only if there is some $d$ with $\langle d, \mathbf{1}\rangle>0$ and yet $V \operatorname{diag}^{*}(d) V^{T} \preceq 0$. Then $D=\operatorname{diag}^{*}(d)$ has the properties in the statement of the lemma.

3.2. Exploiting connections: Results for one-dimensional subspaces. In 1940, Ledermann [21] characterized the one-dimensional subspaces that are recoverable by MTFA. In 1990, Grone, Pierce, and Watkins [15] gave a necessary condition for a subspace to be realizable. In 1993, independently of Ledermann's work, Delorme and Poljak [8] showed that this condition is also sufficient for one-dimensional subspaces. Since we have established that a subspace is recoverable by MTFA if and only if it is realizable, Ledermann's result and Delorme and Poljak's results are equivalent. In this section we translate these equivalent results into the context of the ellipsoid fitting problem, giving a geometric characterization of when it is possible to fit a centered ellipsoid to $k+1$ points in $\mathbb{R}^{k}$.

Delorme and Poljak state their result in terms of the following definition.

Definition 3.3 (Delorme and Poljak [8]). A vector $u \in \mathbb{R}^{n}$ is balanced if

$$
\left|u_{i}\right| \leq \sum_{j \neq i}\left|u_{j}\right| \quad \text { for all } i \in[n] .
$$

If the inequality is strict we say that $u$ is strictly balanced. A subspace $\mathcal{U}$ of $\mathbb{R}^{n}$ is (strictly) balanced if every $u \in \mathcal{U}$ is (strictly) balanced.

In the following, the necessary condition is due to Grone et al. [15] and the sufficient condition is due to Ledermann [21] (in the context of the analysis of MTFA) and Delorme and Poljak [8] (in the context of the facial structure of the elliptope). We state the result only in terms of realizability of a subspace.

THeOREM 3.4. If a subspace $\mathcal{U}$ of $\mathbb{R}^{n}$ is realizable, then it is balanced. If a subspace $\mathcal{U}$ of $\mathbb{R}^{n}$ is balanced and $\operatorname{dim}(\mathcal{U})=1$, then it is realizable.

From Proposition 3.1 we know that whether a subspace is realizable can be determined by deciding whether we can fit an ellipsoid to a particular collection of points. We next develop the analogous geometric interpretation of balanced subspaces.

Copyright $@$ by SIAM. Unauthorized reproduction of this article is prohibited. 
Definition 3.5. A collection of points $v_{1}, v_{2}, \ldots, v_{n} \in \mathbb{R}^{k}$ is in convex position if for each $i \in[n], v_{i}$ lies on the boundary of the convex hull of $\pm v_{1}, \pm v_{2}, \ldots, \pm v_{n}$.

The following lemma makes precise the connection between balanced subspaces and points in convex position.

Lemma 3.6. Suppose $V$ is any $k \times n$ matrix with $\mathcal{N}(V)=\mathcal{U}$. Then $\mathcal{U}$ is balanced if and only if the columns of $V$ are in convex position.

Proof. We defer a detailed proof to Appendix A, giving only the main idea here. We can check if a collection of points is in convex position by checking the feasibility of a system of linear inequalities (given in Appendix A). An application of linear programming duality establishes that certificates of infeasibility of these linear inequalities take the form of elements of $\mathcal{U}$ that are not balanced.

By combining Theorem 3.4 with Lemma 3.6, we are in a position to interpret Theorem 3.4 purely in terms of ellipsoid fitting.

COROLlary 3.7. If there is a centered ellipsoid passing through $v_{1}, v_{2}, \ldots, v_{n} \in$ $\mathbb{R}^{k}$, then they are in convex position. If $v_{1}, v_{2}, \ldots, v_{n} \in \mathbb{R}^{k}$ and $k=n-1$, then there is a centered ellipsoid passing through them.

In this geometric setting the necessary condition is clear-we can only hope to find a centered ellipsoid passing through a collection of points if they are in convex position, i.e., they lie on the boundary of some convex body.

One may wonder for which other $n$ and $k$ (if any) it is the case that there is an ellipsoid passing through any set of $n$ points in $\mathbb{R}^{k}$ that are in convex position (or equivalently that any $(n-k)$-dimensional balanced subspace of $\mathbb{R}^{n}$ is realizable). This is not the case for general $n$ and $k$. For example, let $n \geq\left(\begin{array}{c}k+1 \\ 2\end{array}\right)+1$ and choose $v_{1}, v_{2}, \ldots, v_{n-1}$ on the unit sphere in $\mathbb{R}^{k}$ so that the sphere is the unique centered ellipsoid passing through them. Then choose $v_{n}$ in the interior of the sphere but not in the convex hull of $\pm v_{1}, \pm v_{2}, \ldots, \pm v_{n-1}$. The resulting points are in convex position but there is no centered ellipsoid passing through them.

On a positive note, since there is clearly an ellipsoid passing through any subset of $\mathbb{R}^{1}$ in convex position, we have the following simple addition to Theorem 3.4.

Proposition 3.8. If a subspace $\mathcal{U}$ of $\mathbb{R}^{n}$ is balanced and $\operatorname{dim}(\mathcal{U})=n-1$, then $\mathcal{U}$ is realizable.

4. A sufficient condition for the three problems. In this section we establish a new sufficient condition for a subspace $\mathcal{U}$ of $\mathbb{R}^{n}$ to be realizable and consequently a sufficient condition for $\mathcal{U}$ to be recoverable by MTFA and $\mathcal{U}^{\perp}$ to have the ellipsoid fitting property. Our condition is based on a simple property of a subspace known as coherence.

Given a subspace $\mathcal{U}$ of $\mathbb{R}^{n}$, the coherence of $\mathcal{U}$ is a measure of how close the subspace is to containing any of the elementary unit vectors. This notion was introduced (with a different scaling) by Candès and Recht in their work on low-rank matrix completion [5], although related quantities have played an important role in the analysis of sparse reconstruction problems since the work of Donoho and Huo [10].

Definition 4.1. If $\mathcal{U}$ is a subspace of $\mathbb{R}^{n}$, then the coherence of $\mathcal{U}$ is

$$
\mu(\mathcal{U})=\max _{i \in[n]}\left\|P_{\mathcal{U}} e_{i}\right\|_{2}^{2}
$$

There are a number of equivalent ways to express coherence, some of which we collect here for convenience. Since $P_{\mathcal{U}}^{2}=P_{\mathcal{U}}=I-P_{\mathcal{U}^{\perp}}$,

$$
\mu(\mathcal{U})=\max _{i \in[n]}\left[P_{\mathcal{U}}\right]_{i i}=1-\min _{i \in[n]}\left[P_{\mathcal{U}}\right]_{i i}
$$

Copyright $@$ by SIAM. Unauthorized reproduction of this article is prohibited. 
Coherence also has an interpretation as the square of the worst-case ratio between the infinity norm and the Euclidean norm on a subspace:

$$
\mu(\mathcal{U})=\max _{i \in[n]}\left\|P_{\mathcal{U}} e_{i}\right\|_{2}^{2}=\max _{i \in[n]} \max _{u \in \mathcal{U} \backslash\{0\}} \frac{\left\langle e_{i}, u\right\rangle^{2}}{\|u\|_{2}^{2}}=\max _{u \in \mathcal{U} \backslash\{0\}} \frac{\|u\|_{\infty}^{2}}{\|u\|_{2}^{2}} .
$$

A basic property of coherence is that it satisfies the inequality

$$
\frac{\operatorname{dim}(\mathcal{U})}{n} \leq \mu(\mathcal{U}) \leq 1
$$

for any subspace $\mathcal{U}$ of $\mathbb{R}^{n}[5]$. This holds because $P_{\mathcal{U}}$ has $\operatorname{dim}(\mathcal{U})$ eigenvalues equal to one and the rest equal to zero so that $\operatorname{dim}(\mathcal{U}) / n=\operatorname{tr}\left(P_{\mathcal{U}}\right) / n \leq \max _{i \in[n]}\left[P_{\mathcal{U}}\right]_{i i}=\mu(\mathcal{U})$. The inequality (4.3), together with the definition of coherence, provides useful intuition about the properties of subspaces with low coherence, that is, incoherence. Any subspace with low coherence is necessarily of low dimension and far from containing any of the elementary unit vectors $e_{i}$. As such, any symmetric matrix with incoherent row/column spaces is necessarily of low rank and quite different from being a diagonal matrix.

4.1. Coherence-threshold-type sufficient conditions. In this section we focus on finding the largest possible $\alpha$ such that

$$
\mu(\mathcal{U})<\alpha \Longrightarrow \mathcal{U} \text { is realizable, }
$$

that is, finding the best possible coherence-threshold-type sufficient condition for a subspace to be realizable. Such conditions are of particular interest because the dependence they have on the ambient dimension and the dimension of the subspace is only the mild dependence implied by (4.3). In contrast, existing results (e.g., [8, 20, 19]) about realizability of subspaces hold only for specific combinations of the ambient dimension and the dimension of the subspace.

The following theorem, our main result, gives a sufficient condition for realizability based on a coherence-threshold condition. Furthermore, it establishes that this is the best possible coherence-threshold-type sufficient condition.

TheOREM 4.2. If $\mathcal{U}$ is a subspace of $\mathbb{R}^{n}$ and $\mu(\mathcal{U})<1 / 2$, then $\mathcal{U}$ is realizable. On the other hand, given any $\alpha>1 / 2$, there is a subspace $\mathcal{U}$ with $\mu(\mathcal{U})=\alpha$ that is not realizable.

Proof. We give the main idea of the proof, deferring some details to Appendix A. Instead of proving that there is some $Y \in \mathcal{F}_{\mathcal{U}}=\{Y \succeq 0: \mathcal{N}(Y) \supseteq \mathcal{U}\}$ such that $Y_{i i}=1$ for $i \in[n]$, it suffices to choose a convex cone $\mathcal{K}$ that is an inner approximation to $\mathcal{F}_{\mathcal{U}}$ and establish that there is some $Y \in \mathcal{K}$ such that $Y_{i i}=1$ for $i \in[n]$. One natural choice is to take $\mathcal{K}=\left\{P_{\mathcal{U} \perp} \operatorname{diag}^{*}(\lambda) P_{\mathcal{U}^{\perp}}: \lambda \geq 0\right\}$, which is clearly contained in $\mathcal{F}_{\mathcal{U}}$. Note that there is some $Y \in \mathcal{K}$ such that $Y_{i i}=1$ for all $i \in[n]$ if and only if there is $\lambda \geq 0$ such that

$$
\operatorname{diag}\left(P_{\mathcal{U}^{\perp}} \operatorname{diag}^{*}(\lambda) P_{\mathcal{U}^{\perp}}\right)=\mathbf{1}
$$

The rest of the proof of the sufficient condition involves showing that if $\mu(\mathcal{U})<1 / 2$, then such a nonnegative $\lambda$ exists. We establish this in Lemma A.1.

Now let us construct, for any $\alpha>1 / 2$, a subspace with coherence $\alpha$ that is not realizable. Let $\mathcal{U}$ be the subspace of $\mathbb{R}^{2}$ spanned by $u=(\sqrt{\alpha}, \sqrt{1-\alpha})$. Then $\mu(\mathcal{U})=\max \{\alpha, 1-\alpha\}=\alpha$ and yet by Theorem 3.4, $\mathcal{U}$ is not realizable because $u$ is not balanced. 
Comparison with results on sparse and low-rank decompositions. In [6, Corollary 3] a sufficient condition is given under which a convex program related to MTFA can successfully decompose the sum of an unknown sparse matrix $S^{\star}$ and an unknown low-rank matrix $L^{\star}$. The condition is that $\operatorname{deg}_{\max }\left(S^{\star}\right) \operatorname{inc}\left(L^{\star}\right)<1 / 12$, where $\operatorname{deg}_{\max }\left(S^{\star}\right)$ is the maximum number of nonzero entries per row/column of $S^{\star}$ and, for a symmetric matrix $L^{\star}$ with column space $\mathcal{U}, \operatorname{inc}\left(L^{\star}\right)=\sqrt{\mu(\mathcal{U})}$ is the square root of the coherence as defined in the present paper. As such, Chandrasekaran et al. show that the convex program they analyze successfully decomposes the sum of a diagonal matrix and a symmetric matrix with column space $\mathcal{U}$ as long as $1 \cdot \sqrt{\mu(\mathcal{U})}<1 / 12$ or equivalently $\mu(\mathcal{U})<1 / 144$. By comparison, in this paper both the convex program we consider and our analysis of that convex program exploit the assumptions that the sparse matrix is diagonal and that the low-rank matrix is positive semidefinite. This allows us to obtain the much more refined sufficient condition $\mu(\mathcal{U})<1 / 2$.

We now establish two corollaries of our coherence-threshold-type sufficient condition for realizability. These corollaries can be thought of as reinterpretations of the coherence inequality $\mu(\mathcal{U})<1 / 2$ in terms of other natural quantities.

An ellipsoid-fitting interpretation. With the aid of Proposition 3.1 we reinterpret our coherence-threshold-type sufficient condition as a sufficient condition on a set of points in $\mathbb{R}^{k}$ that ensures there is a centered ellipsoid passing through them. The condition involves "sandwiching" the points between two ellipsoids (that depend on the points). Indeed, given $0<\beta<1$ and points $v_{1}, v_{2}, \ldots, v_{n} \in \mathbb{R}^{k}$ that span $\mathbb{R}^{k}$, we define the ellipsoid

$$
\mathcal{E}_{\beta}\left(v_{1}, \ldots, v_{n}\right)=\left\{x \in \mathbb{R}^{k}: x^{T}\left(\sum_{j=1}^{n} v_{j} v_{j}^{T}\right)^{-1} x \leq \beta\right\} .
$$

Definition 4.3. Given $0<\beta<1$ the points $v_{1}, v_{2}, \ldots, v_{n}$ satisfy the $\beta$-sandwich condition if $v_{1}, v_{2}, \ldots, v_{n}$ span $\mathbb{R}^{k}$ and

$$
\left\{v_{1}, v_{2}, \ldots, v_{n}\right\} \subset \mathcal{E}_{1}\left(v_{1}, \ldots, v_{n}\right) \backslash \mathcal{E}_{\beta}\left(v_{1}, \ldots, v_{n}\right) .
$$

The intuition behind this definition (illustrated in Figure 4.1) is that if the points satisfy the $\beta$-sandwich condition for $\beta$ close to one, then they are confined to a thin ellipsoidal shell that is adapted to their position. One might expect that it is "easier" to fit an ellipsoid to points that are confined in this way. Indeed this is the case.

Corollary 4.4. If $v_{1}, v_{2}, \ldots, v_{n} \in \mathbb{R}^{k}$ satisfy the $1 / 2$-sandwich condition, then there is a centered ellipsoid passing through $v_{1}, v_{2}, \ldots, v_{n}$.

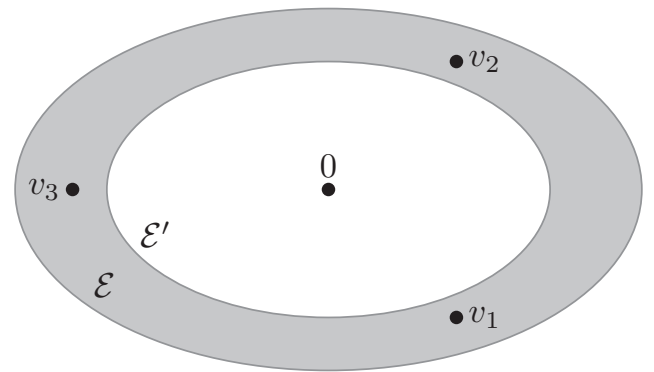

FIG. 4.1. The ellipsoids shown are $\mathcal{E}=\mathcal{E}_{1}\left(v_{1}, v_{2}, v_{3}\right)$ and $\mathcal{E}^{\prime}=\mathcal{E}_{1 / 2}\left(v_{1}, v_{2}, v_{3}\right)$. There is an ellipsoid passing through $v_{1}, v_{2}$, and $v_{3}$ because the points are sandwiched between $\mathcal{E}$ and $\mathcal{E}^{\prime}$. 
Proof. Let $V$ be the $k \times n$ matrix with columns given by the $v_{i}$, and let $\mathcal{U}$ be the $(n-k)$-dimensional nullspace of $V$. Then the orthogonal projection onto the row space of $V$ is $P_{\mathcal{U}^{\perp}}$ and can be written as

$$
P_{\mathcal{U}^{\perp}}=V^{T}\left(V V^{T}\right)^{-1} V
$$

Our assumption that the points satisfy the $1 / 2$-sandwich condition is equivalent to assuming that $1 / 2<\left[P_{\mathcal{U}^{\perp}}\right]_{i i} \leq 1$ for all $i \in[n]$, which by (4.1) is equivalent to $\mu(\mathcal{U})<1 / 2$. From Theorem 4.2 we know that $\mu(\mathcal{U})<1 / 2$ implies that $\mathcal{U}$ is realizable. Invoking Proposition 3.1 we then conclude that there is a centered ellipsoid passing through $v_{1}, v_{2}, \ldots, v_{n}$.

A balance interpretation. In section 3.2 we saw that if a subspace $\mathcal{U}$ is realizable, every $u \in \mathcal{U}$ is balanced. The sufficient condition of Theorem 4.2 can be expressed in terms of a balance condition on the elementwise square of the elements of a subspace. (In what follows $u \circ u$ denotes the elementwise square of a vector in $\mathbb{R}^{n}$.)

Lemma 4.5. If $\mathcal{U}$ is a subspace of $\mathbb{R}^{n}$, then

$$
\begin{aligned}
\mu(\mathcal{U})<1 / 2 & \Longleftrightarrow u \circ u \text { is strictly balanced for all } u \in \mathcal{U}, \\
& \Longrightarrow \mathcal{U} \text { is strictly balanced. }
\end{aligned}
$$

Proof. By the characterization of $\mu(\mathcal{U})$ in $(4.2)$

$$
\mu(\mathcal{U})<1 / 2 \Longleftrightarrow \max _{u \in \mathcal{U} \backslash\{0\}}\|u\|_{\infty}^{2} /\|u\|_{2}^{2}<1 / 2,
$$

which in turn is equivalent to $u_{i}^{2}<\sum_{j \neq i} u_{j}^{2}$ for all $i \in[n]$ and all $u \in \mathcal{U}$, i.e., $u \circ u$ is strictly balanced for all $u \in \mathcal{U}$.

Suppose $u \circ u$ is strictly balanced for all $u \in \mathcal{U}$. Then for all $i \in[n]$ and all $u \in \mathcal{U}$

$$
\left|u_{i}\right|<\left(\sum_{j \neq i} u_{j}^{2}\right)^{1 / 2} \leq \sum_{j \neq i}\left|u_{j}\right|,
$$

i.e., $\mathcal{U}$ is strictly balanced. (Here we have used the fact that $\|x\|_{2} \leq\|x\|_{1}$ for any $x \in \mathbb{R}^{n}$.)

With this relationship established we can express both the known necessary condition and our sufficient condition for a subspace to be realizable in terms of the notion of balance. The necessary condition is restated from Theorem 3.4; the sufficient condition follows by combining Theorem 4.2 and Lemma 4.5.

Corollary 4.6. If a subspace $\mathcal{U}$ is realizable, then every $u \in \mathcal{U}$ is balanced. If $u \circ u$ is strictly balanced for every $u \in \mathcal{U}$, then the subspace $\mathcal{U}$ is realizable.

Remark. Suppose $\mathcal{U}=\operatorname{span}\{u\}$ is a one-dimensional subspace of $\mathbb{R}^{n}$. We have just established that if $u \circ u$ is strictly balanced, then $\mathcal{U}$ is realizable and so (by Theorem 3.4) $u$ must be balanced, a fact we proved directly in Lemma 4.5.

4.2. Examples. To gain more intuition for what Theorem 4.2 means, we consider its implications in two particular cases. First, we compare the characterization of when it is possible to fit an ellipsoid to $k+1$ points in $\mathbb{R}^{k}$ (Corollary 3.7 ) with the specialization of our sufficient condition to this case (Corollary 4.4). This comparison provides some geometric insight into how conservative our sufficient condition is. Second, we investigate the coherence properties of suitably random subspaces. This provides intuition about whether $\mu(\mathcal{U})<1 / 2$ is a very restrictive condition. In particular, we establish that "most" subspaces of $\mathbb{R}^{n}$ with dimension bounded above by $(1 / 2-\epsilon) n$ are realizable. 


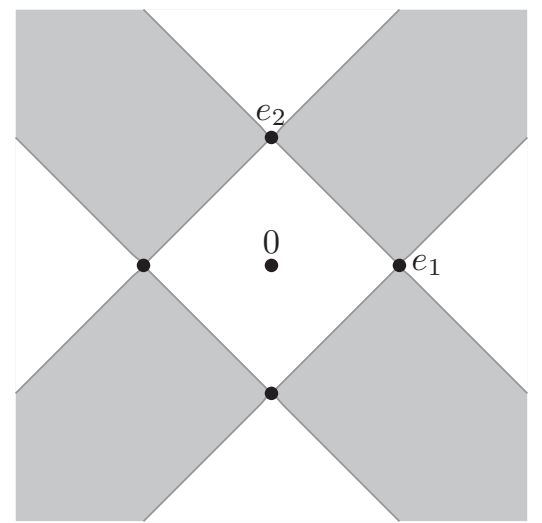

(a) The shaded set is $R$, those points $w$ for which we can fit an ellipsoid through $w$ and the standard basis vectors.

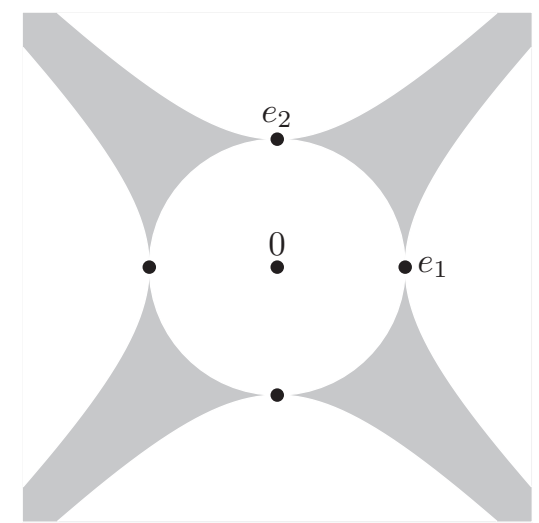

(b) The shaded set is $R^{\prime}$, those points $w$ such that $w, e_{1}$, and $e_{2}$ satisify the condition of Corollary 4.4.

FIG. 4.2. Comparing our sufficient condition (Corollary 4.4) with the characterization (Corollary 3.7$)$ in the case of fitting an ellipsoid to $k+1$ points in $\mathbb{R}^{k}$.

Fitting an ellipsoid to $k+1$ points in $\mathbb{R}^{k}$. Recall that the result of Ledermann and Delorme and Poljak, interpreted in terms of ellipsoid fitting, tells us that we can fit an ellipsoid to $k+1$ points $v_{1}, \ldots, v_{k+1} \in \mathbb{R}^{k}$ if and only if those points are in convex position (see Corollary 3.7). We now compare this characterization with the $1 / 2$-sandwich condition, which is sufficient by Corollary 4.4 .

Without loss of generality we assume that $k$ of the points are $e_{1}, \ldots, e_{k}$, the standard basis vectors, and compare the conditions by considering the set of locations of the $(k+1)$ st point $w \in \mathbb{R}^{k}$ for which we can fit an ellipsoid through all $k+1$ points. Corollary 3.7 gives a characterization of this region as

$$
R=\left\{w \in \mathbb{R}^{k}: \sum_{j=1}^{k}\left|w_{j}\right| \geq 1,\left|w_{i}\right|-\sum_{j \neq i}\left|w_{j}\right| \leq 1 \quad \text { for } i \in[k]\right\},
$$

which is shown in Figure 4.2(a) in the case $k=2$. The set of $w$ such that $w, e_{1}, \ldots, e_{n}$ satisfy the $1 / 2$-sandwich condition can be written as

$$
\begin{aligned}
R^{\prime} & =\left\{w \in \mathbb{R}^{k}: w^{T}\left(I+w w^{T}\right)^{-1} w>1 / 2, e_{i}^{T}\left(I+w w^{T}\right)^{-1} e_{i}>1 / 2 \quad \text { for } i \in[k]\right\} \\
& =\left\{w \in \mathbb{R}^{k}: \sum_{j=1}^{k} w_{j}^{2}>1, w_{i}^{2}-\sum_{j \neq i} w_{j}^{2}<1 \quad \text { for } i \in[k]\right\}
\end{aligned}
$$

where the second equality holds because $\left(I+w w^{T}\right)^{-1}=I-\frac{w w^{T}}{1+w^{T} w}$. The region $R^{\prime}$ is shown in Figure 4.2(b) in the case $k=2$. It is clear that $R^{\prime} \subseteq R$.

Realizability of random subspaces. Suppose $\mathcal{U}$ is a subspace generated by taking the column space of an $n \times r$ matrix with independent and identically distributed standard Gaussian entries. For what values of $r$ and $n$ does such a subspace have $\mu(\mathcal{U})<1 / 2$ with high probability, i.e., satisfy our sufficient condition for being realizable?

The following result essentially shows that for large $n$, "most" subspaces of dimension at most $(1 / 2-\epsilon) n$ are realizable. This suggests that MTFA is a very good heuristic 
for diagonal and low-rank decomposition problems in the high-dimensional setting. Indeed "most" subspaces of dimension up to one half the ambient dimension-hardly just low-dimensional subspaces - are recoverable by MTFA.

Proposition 4.7. Let $0<\epsilon<1 / 2$ be a constant and suppose $n>6 /\left(\epsilon^{2}-2 \epsilon^{3}\right)$. There are positive constants $\bar{c}, \tilde{c}$, (depending only on $\epsilon$ ) such that if $\mathcal{U}$ is a random $(1 / 2-\epsilon) n$ dimensional subspace of $\mathbb{R}^{n}$, then

$$
\operatorname{Pr}[\mathcal{U} \text { is realizable }] \geq 1-\bar{c} \sqrt{n} e^{-\tilde{c} n} .
$$

We provide a proof of this result in Appendix A. The main idea is that the coherence of a random $r$-dimensional subspace of $\mathbb{R}^{n}$ is the maximum of $n$ random variables that concentrate around their mean of $r / n$ for large $n$.

To illustrate the result, we consider the case where $\epsilon=1 / 4$ and $n>192$. Then (by examining the proof in Appendix A) we see that we can take $\tilde{c}=1 / 24$ and $\bar{c}=24 / \sqrt{3 \pi} \approx 7.8$. Hence if $n>192$ and $\mathcal{U}$ is a random $n / 4$ dimensional subspace of $\mathbb{R}^{n}$ we have that

$$
\operatorname{Pr}[\mathcal{U} \text { is realizable }] \geq 1-7.8 \sqrt{n} e^{-n / 24} \text {. }
$$

5. Tractable block-diagonal and low-rank decompositions and related problems. In this section we generalize our results to the analogue of MTFA for block-diagonal and low-rank decompositions. Mimicking our earlier development, we relate the analysis of this variant of MTFA to the facial structure of a variant of the elliptope and a generalization of the ellipsoid fitting problem. The key point is that these problems all possess additional symmetries that, once taken into account, essentially allow us to reduce our analysis to cases already considered in sections 3 and 4 .

Throughout this section, let $\mathcal{P}$ be a fixed partition of $\{1,2, \ldots, n\}$. We say a matrix is $\mathcal{P}$-block-diagonal if it is zero except for the principal submatrices indexed by the elements of $\mathcal{P}$. We denote by blkdiag ${ }_{\mathcal{P}}$ the map that takes an $n \times n$ matrix and maps it to the principal submatrices indexed by $\mathcal{P}$. Its adjoint, denoted blkdiag ${ }_{\mathcal{P}}^{*}$, takes a tuple of symmetric matrices $\left(X_{\mathcal{I}}\right)_{\mathcal{I} \in \mathcal{P}}$ and produces an $n \times n$ matrix that is $\mathcal{P}$-block-diagonal with blocks given by the $X_{\mathcal{I}}$.

We now describe the analogues of MTFA, ellipsoid fitting, and the problem of determining the facial structure of the elliptope.

Block minimum trace factor analysis. If $X=B^{\star}+L^{\star}$, where $B^{\star}$ is $\mathcal{P}$-blockdiagonal and $L^{\star} \succeq 0$ is low rank, the obvious analogue of MTFA is the semidefinite program

$$
\underset{B, L}{\operatorname{minimize}} \operatorname{tr}(L) \quad \text { subject to }\left\{\begin{array}{l}
X=B+L \\
L \succeq 0, \\
B \text { is } \mathcal{P} \text {-block-diagonal, }
\end{array}\right.
$$

which we call block minimum trace factor analysis (BMTFA). A straightforward modification of the Della Riccia and Shapiro argument for MTFA [7] shows that if BMTFA is feasible it has a unique optimal solution.

Definition 5.1. A subspace $\mathcal{U}$ of $\mathbb{R}^{n}$ is recoverable by BMTFA if for every $B^{\star}$ that is $\mathcal{P}$-block-diagonal and every positive semidefinite $L^{\star}$ with column space $\mathcal{U}$, $\left(B^{\star}, L^{\star}\right)$ is the optimum of BMTFA with input $X=B^{\star}+L^{\star}$.

Faces of the $\mathcal{P}$-elliptope. Just as MTFA is related to the facial structure of the elliptope, BMTFA is related to the facial structure of the spectrahedron

$$
\mathcal{E}_{\mathcal{P}}=\left\{Y \succeq 0: \operatorname{blkdiag}_{\mathcal{P}}(Y)=(I, I, \ldots, I)\right\} .
$$

Copyright $@$ by SIAM. Unauthorized reproduction of this article is prohibited. 
We refer to $\mathcal{E}_{\mathcal{P}}$ as the $\mathcal{P}$-elliptope. We extend the definition of a realizable subspace to this context.

Definition 5.2. A subspace $\mathcal{U}$ of $\mathbb{R}^{n}$ is $\mathcal{P}$-realizable if there is some $Y \in \mathcal{E}_{\mathcal{P}}$ such that $\mathcal{N}(Y) \supseteq \mathcal{U}$.

Generalized ellipsoid fitting. To describe the $\mathcal{P}$-ellipsoid fitting problem we first introduce some convenient notation. If $\mathcal{I} \subset[n]$ we write

$$
S^{\mathcal{I}}=\left\{x \in \mathbb{R}^{n}:\|x\|_{2}=1, x_{j}=0 \text { if } j \notin \mathcal{I}\right\}
$$

for the intersection of the unit sphere with the coordinate subspace indexed by $\mathcal{I}$.

Suppose $v_{1}, v_{2}, \ldots, v_{n} \in \mathbb{R}^{k}$ is a collection of points and $V$ is the $k \times n$ matrix with columns given by the $v_{i}$. Noting that $S^{\{i\}}=\left\{-e_{i}, e_{i}\right\}$, and thinking of $V$ as a linear map from $\mathbb{R}^{n}$ to $\mathbb{R}^{k}$, we see that the ellipsoid fitting problem is to find an ellipsoid in $\mathbb{R}^{k}$ with boundary containing $\cup_{i \in[n]} V\left(S^{\{i\}}\right)$, i.e., the collection of points $\pm v_{1}, \ldots, \pm v_{n}$. The $\mathcal{P}$-ellipsoid fitting problem is then to find an ellipsoid in $\mathbb{R}^{k}$ with boundary containing $\cup_{\mathcal{I} \in \mathcal{P}} V\left(S^{\mathcal{I}}\right)$, i.e., the collection of ellipsoids $V\left(S^{\mathcal{I}}\right)$.

The generalization of the ellipsoid fitting property of a subspace is as follows.

Definition 5.3. A $k$-dimensional subspace $\mathcal{V}$ of $\mathbb{R}^{n}$ has the $\mathcal{P}$-ellipsoid fitting property if there is a $k \times n$ matrix $V$ with row space $\mathcal{V}$ such that there is a centered ellipsoid in $\mathbb{R}^{k}$ with boundary containing $\cup_{\mathcal{I} \in \mathcal{P}} V\left(S^{\mathcal{I}}\right)$.

5.1. Relating the generalized problems. The facial structure of the $\mathcal{P}$ elliptope, BMTFA, and the $\mathcal{P}$-ellipsoid fitting problem are related by the following result, the proof of which is omitted as it is almost identical to that of Proposition 3.1.

Proposition 5.4. Let $\mathcal{U}$ be a subspace of $\mathbb{R}^{n}$. Then the following are equivalent:

1. $\mathcal{U}$ is recoverable by BMTFA.

2. $\mathcal{U}$ is $\mathcal{P}$-realizable.

3. $\mathcal{U}^{\perp}$ has the $\mathcal{P}$-ellipsoid fitting property.

The following lemma is the analogue of Lemma 3.2. It describes certificates that a subspace $\mathcal{U}$ is not $\mathcal{P}$-realizable. Again the proof is almost identical to that of Lemma 3.2 , so we omit it.

Lemma 5.5. A subspace $\mathcal{U}$ of $\mathbb{R}^{n}$ is not $\mathcal{P}$-realizable if and only if there is a $\mathcal{P}$-block-diagonal matrix $B$ such that $\operatorname{tr}(B)>0$ and $v^{T} B v \leq 0$ for all $v \in \mathcal{U}^{\perp}$.

For the sake of brevity, in what follows we only discuss the problem of whether $\mathcal{U}$ is $\mathcal{P}$-realizable without explicitly translating the results into the context of the other two problems.

5.2. Symmetries of the $\mathcal{P}$-elliptope. We now consider the symmetries of the $\mathcal{P}$-elliptope. Our motivation for doing so is that it allows us to partition subspaces into classes for which either all elements are $\mathcal{P}$-realizable or none of the elements are $\mathcal{P}$-realizable.

It is clear that the $\mathcal{P}$-elliptope is invariant under conjugation by $\mathcal{P}$-block-diagonal orthogonal matrices. Let $G_{\mathcal{P}}$ denote this subgroup of the group of $n \times n$ orthogonal matrices. There is a natural action of $G_{\mathcal{P}}$ on subspaces of $\mathbb{R}^{n}$ defined as follows. If $P \in G_{\mathcal{P}}$ and $\mathcal{U}$ is a subspace of $\mathbb{R}^{n}$, then $P \cdot \mathcal{U}$ is the image of the subspace $\mathcal{U}$ under the map $P$. (It is straightforward to check that this is a well-defined group action.) If there exists some $P \in G_{\mathcal{P}}$ such that $P \cdot \mathcal{U}=\mathcal{U}^{\prime}$, then we write $\mathcal{U} \sim \mathcal{U}^{\prime}$ and say that $\mathcal{U}$ and $\mathcal{U}^{\prime}$ are equivalent. We care about this equivalence relation on subspaces because the property of being $\mathcal{P}$-realizable is really a property of the corresponding equivalence classes.

Proposition 5.6. Suppose $\mathcal{U}$ and $\mathcal{U}^{\prime}$ are subspaces of $\mathbb{R}^{n}$. If $\mathcal{U} \sim \mathcal{U}^{\prime}$, then $\mathcal{U}$ is $\mathcal{P}$-realizable if and only if $\mathcal{U}^{\prime}$ is $\mathcal{P}$-realizable. 
Proof. If $\mathcal{U}$ is $\mathcal{P}$-realizable there is $Y \in \mathcal{E}_{\mathcal{P}}$ such that $Y u=0$ for all $u \in \mathcal{U}$. Suppose $\mathcal{U}^{\prime}=P \cdot \mathcal{U}$ for some $P \in G_{\mathcal{P}}$ and let $Y^{\prime}=P Y P^{T}$. Then $Y^{\prime} \in \mathcal{E}_{\mathcal{P}}$ and $Y^{\prime}(P u)=\left(P Y P^{T}\right)(P u)=0$ for all $u \in \mathcal{U}$. By the definition of $\mathcal{U}^{\prime}$ it is then the case that $Y^{\prime} u^{\prime}=0$ for all $u^{\prime} \in \mathcal{U}^{\prime}$. Hence $\mathcal{U}^{\prime}$ is $\mathcal{P}$-realizable. The converse clearly also holds.

5.3. Exploiting symmetries: Relating realizability and $\mathcal{P}$-realizability. For a subspace of $\mathbb{R}^{n}$, we now consider how the notions of $\mathcal{P}$-realizability and realizability (i.e., $[n]$-realizability) relate to each other. Since $\mathcal{E}_{\mathcal{P}} \subset \mathcal{E}_{n}$, if $\mathcal{U}$ is $\mathcal{P}$-realizable, it is certainly also realizable. While the converse does not hold, we can establish the following partial converse, which we subsequently use to extend our analysis from sections 3 and 4 to the present setting.

Theorem 5.7. A subspace $\mathcal{U}$ of $\mathbb{R}^{n}$ is $\mathcal{P}$-realizable if and only if $\mathcal{U}^{\prime}$ is realizable for every $\mathcal{U}^{\prime}$ such that $\mathcal{U}^{\prime} \sim \mathcal{U}$.

Proof. We note that one direction of the proof is obvious since $\mathcal{P}$-realizability implies realizability. It remains to show that if $\mathcal{U}$ is not $\mathcal{P}$-realizable, then there is some $\mathcal{U}^{\prime}$ equivalent to $\mathcal{U}$ that is not realizable.

Recall from Lemma 5.5 that if $\mathcal{U}$ is not $\mathcal{P}$-realizable there is some $\mathcal{P}$-block-diagonal $X$ with positive trace such that $v^{T} X v \leq 0$ for all $v \in \mathcal{U}^{\perp}$. Since $X$ is $\mathcal{P}$-blockdiagonal there is some $P \in G_{\mathcal{P}}$ such that $P X P^{T}$ is diagonal. Since conjugation by orthogonal matrices preserves eigenvalues, $\operatorname{tr}\left(P X P^{T}\right)=\operatorname{tr}(X)>0$. Furthermore, $v^{T}\left(P X P^{T}\right) v=\left(P^{T} v\right)^{T} X\left(P^{T} v\right) \leq 0$ for all $P^{T} v \in \mathcal{U}^{\perp}$. Hence $w^{T}\left(P X P^{T}\right) w \geq 0$ for all $w \in P \cdot \mathcal{U}^{\perp}=(P \cdot \mathcal{U})^{\perp}$. By Lemma 3.2,PXP $P^{T}$ is a certificate that $P \cdot \mathcal{U}$ is not realizable, completing the proof.

The power of Theorem 5.7 lies in its ability to turn any condition for a subspace to be realizable into a condition for the subspace to be $\mathcal{P}$-realizable by appropriately symmetrizing the condition with respect to the action of $G_{\mathcal{P}}$. We now illustrate this approach by generalizing Theorem 3.4 and our coherence-based condition (Theorem 4.2) for a subspace to be $\mathcal{P}$-realizable. In each case we first define an appropriately symmetrized version of the original condition. The natural symmetrized version of the notion of balance is as follows.

Definition 5.8. A vector $u \in \mathbb{R}^{n}$ is $\mathcal{P}$-balanced if for all $\mathcal{I} \in \mathcal{P}$

$$
\left\|u_{\mathcal{I}}\right\|_{2} \leq \sum_{\mathcal{J} \in \mathcal{P} \backslash\{\mathcal{I}\}}\left\|u_{\mathcal{J}}\right\|_{2}
$$

We next define the appropriately symmetrized analogue of coherence. Just as coherence measures how far a subspace is from any one-dimensional coordinate subspace, $\mathcal{P}$-coherence measures how far a subspace is from any of the coordinate subspaces indexed by elements of $\mathcal{P}$.

DeFinition 5.9. The $\mathcal{P}$-coherence of a subspace $\mathcal{U}$ of $\mathbb{R}^{n}$ is

$$
\mu_{\mathcal{P}}(\mathcal{U})=\max _{\mathcal{I} \in \mathcal{P}} \max _{x \in S^{\mathcal{I}}}\left\|P_{\mathcal{U}} x\right\|_{2}^{2}
$$

Just as the coherence of $\mathcal{U}$ can be computed by taking the maximum diagonal element of $P_{\mathcal{U}}$, it is straightforward to verify that the $\mathcal{P}$-coherence of $\mathcal{U}$ can be computed by taking the maximum of the spectral norms of the principal submatrices $\left[P_{\mathcal{U}}\right]_{\mathcal{I}}$ indexed by $\mathcal{I} \in \mathcal{P}$.

We now use Theorem 5.7 to establish the natural generalization of Theorem 3.4.

Corollary 5.10. If a subspace $\mathcal{U}$ of $\mathbb{R}^{n}$ is $\mathcal{P}$-realizable, then every element of $\mathcal{U}$ is $\mathcal{P}$-balanced. If $\mathcal{U}=\operatorname{span}\{u\}$ is one-dimensional, then $\mathcal{U}$ is $\mathcal{P}$-realizable if and only if $u$ is $\mathcal{P}$-balanced. 
Proof. If there is $u \in \mathcal{U}$ that is not $\mathcal{P}$-balanced, then there is $P \in G_{\mathcal{P}}$ such that $P u$ is not balanced. (Choose $P$ so that it rotates each $u_{\mathcal{I}}$ until it has only one nonzero entry.) But then $P \cdot \mathcal{U}$ is not realizable and so $\mathcal{U}$ is not $\mathcal{P}$-realizable.

For the converse, we first show that if a vector is $\mathcal{P}$-balanced, then it is balanced. Let $\mathcal{I} \in \mathcal{P}$, and consider $i \in \mathcal{I}$. Then since $u$ is $\mathcal{P}$-balanced,

$$
2\left|u_{i}\right| \leq 2\left\|u_{\mathcal{I}}\right\|_{2} \leq \sum_{\mathcal{J} \in \mathcal{P}}\left\|u_{\mathcal{J}}\right\|_{2} \leq \sum_{i=1}^{n}\left|u_{i}\right|
$$

and so $u$ is balanced.

Now suppose $\mathcal{U}=\operatorname{span}\{u\}$ is one-dimensional and $u$ is $\mathcal{P}$-balanced. Since $u$ is $\mathcal{P}$ balanced it follows that $P u$ is $\mathcal{P}$-balanced (and hence balanced) every $P \in G_{\mathcal{P}}$. Then by Theorem $3.4 \operatorname{span}\{P u\}$ is realizable for every $P \in G_{\mathcal{P}}$. Hence by Theorem 5.7, $\mathcal{U}$ is $\mathcal{P}$-realizable.

Similarly, with the aid of Theorem 5.7 we can write a $\mathcal{P}$-coherence-threshold condition that is a sufficient condition for a subspace to be $\mathcal{P}$-realizable. The following is a natural generalization of Theorem 4.2.

Corollary 5.11. If $\mu_{\mathcal{P}}(\mathcal{U})<1 / 2$, then $\mathcal{U}$ is $\mathcal{P}$-realizable.

Proof. By examining the constraints in the variational definitions of $\mu(\mathcal{U})$ and $\mu_{\mathcal{P}}(\mathcal{U})$ we see that $\mu(\mathcal{U}) \leq \mu_{\mathcal{P}}(\mathcal{U})$. Consequently if $\mu_{\mathcal{P}}(\mathcal{U})<1 / 2$ it follows from Theorem 4.2 that $\mathcal{U}$ is realizable. Since $\mu_{\mathcal{P}}$ is invariant under the action of $G_{\mathcal{P}}$ on subspaces we can apply Theorem 5.7 to complete the proof.

6. Conclusions. We established a link between three problems of independent interest: deciding whether there is a centered ellipsoid passing through a collection of points, understanding the structure of the faces of the elliptope, and deciding which pairs of diagonal and low-rank matrices can be recovered from their sum using a tractable semidefinite-programming-based heuristic, namely MTFA. We provided a simple sufficient condition, based on the notion of the coherence of a subspace, which ensures the success of MTFA and showed that this is the best possible coherencethreshold-type sufficient condition for this problem. Finally we gave natural generalizations of our results to the problem of analyzing tractable block-diagonal and low-rank decompositions, showing how the symmetries of this problem allow us to reduce much of the analysis to the original case of diagonal and low-rank decompositions.

\section{Appendix A. Additional proofs.}

A.1. Proof of Lemma 3.6. We first establish Lemma 3.6, which gives an interpretation of the balance condition in terms of ellipsoid fitting.

Proof. The proof is a fairly straightforward application of linear programming duality. Throughout let $V$ be the $k \times n$ matrix with columns given by the $v_{i}$. The point $v_{i} \in \mathbb{R}^{k}$ is on the boundary of the convex hull of $\pm v_{1}, \ldots, \pm v_{n}$ if and only if there exists $x \in \mathbb{R}^{k}$ such that $\left\langle x, v_{i}\right\rangle=1$ and $\left|\left\langle x, v_{j}\right\rangle\right| \leq 1$ for all $j \neq i$. Equivalently, the following linear program (which depends on $i$ ) is feasible:

$$
\underset{x}{\operatorname{minimize}}\langle 0, x\rangle \text { subject to }\left\{\begin{array}{c}
v_{i}^{T} x=1, \\
\left|v_{j}^{T} x\right| \leq 1 \text { for all } j \neq i .
\end{array}\right.
$$

Suppose there is some $i$ such that $v_{i}$ is in the interior of $\operatorname{conv}\left\{ \pm v_{1}, \ldots, \pm v_{n}\right\}$. Then 
(A.1) is not feasible so the dual linear program (which depends on $i$ )

$$
\underset{u}{\operatorname{maximize}} u_{i}-\sum_{j \neq i}\left|u_{j}\right| \quad \text { subject to } \quad V u=0
$$

is unbounded. This is the case if and only if there is some $u$ in the nullspace of $V$ such that $u_{i}>\sum_{j \neq i}\left|u_{j}\right|$. If such a $u$ exists, then it is certainly the case that $\left|u_{i}\right| \geq u_{i}>\sum_{j \neq i}\left|u_{j}\right|$ and so $u$ is not balanced.

Conversely, if $u$ is in the nullspace of $V$ and $u$ is not balanced, then either $u$ or $-u$ satisfies $u_{i}>\sum_{j \neq i}\left|u_{j}\right|$ for some $i$. Hence the linear program (A.2) associated with the index $i$ is unbounded and so the corresponding linear program (A.1) is infeasible. It follows that $v_{i}$ is in the interior of the convex hull of $\pm v_{1}, \ldots, \pm v_{n}$.

A.2. Completing the proof of Theorem 4.2. We now complete the proof of Theorem 4.2 by establishing the following result about the existence of a nonnegative solution to the linear system (4.4).

Lemma A.1. If $\mu(\mathcal{U})<1 / 2$, then $\operatorname{diag}\left(P_{\mathcal{U}^{\perp}} \operatorname{diag}^{*}(\lambda) P_{\mathcal{U}^{\perp}}\right)=\mathbf{1}$ has a nonnegative solution $\lambda$.

Proof. We note that the linear system $\operatorname{diag}\left(P_{\mathcal{U}^{\perp}} \operatorname{diag}^{*}(\lambda) P_{\mathcal{U}^{\perp}}\right)=\mathbf{1}$ can be rewritten as $P_{\mathcal{U}^{\perp}} \circ P_{\mathcal{U}^{\perp}} \lambda=\mathbf{1}$, where $\circ$ denotes the entrywise product of matrices. As such, we need to show that $P_{\mathcal{U}_{\perp}} \circ P_{\mathcal{U}_{\perp}}$ is invertible and $\left(P_{\mathcal{U}_{\perp}} \circ P_{\mathcal{U}^{\perp}}\right)^{-1} \mathbf{1} \geq 0$. To do so, we appeal to the following (slight restatement) of a theorem of Walters [31] regarding positive solutions to certain linear systems.

Theorem A.2 (Walters [31]). Suppose $A$ is a square matrix with nonnegative entries and positive diagonal entries. Let $D$ be a diagonal matrix with $D_{i i}=A_{i i}$ for all $i$. If $y>0$ and $2 y-A D^{-1} y>0$, then $A$ is invertible and $A^{-1} y>0$.

For simplicity of notation let $P=P_{\mathcal{U}^{\perp}}$. We apply Theorem A.2 with $A=P \circ P$ and $y=\mathbf{1}$ and so need to check that $(P \circ P) D^{-1} \mathbf{1}<2 \mathbf{1}$, where $D$ is the diagonal matrix with $D_{i i}=P_{i i}^{2}$ for $i \in[n]$.

Since $P^{2}=P$ it follows that for all $i \in[n], \sum_{j=1}^{n} P_{i j}^{2}=\left[P^{2}\right]_{i i}=P_{i i}$. Our assumption that $\mu(\mathcal{U})<1 / 2$ implies that $\min _{i \in[n]} P_{i i}>1 / 2$ and so $D_{i i}=P_{i i}^{-2}<4$ and $P_{i i}-P_{i i}^{2}<1 / 4$ for all $i \in[n]$. Hence

$\left[(P \circ P) D^{-1} \mathbf{1}\right]_{i}=\sum_{j=1}^{n} P_{i j}^{2} D_{j j}^{-1}=1+\sum_{j \neq i} P_{i j}^{2} D_{j j}^{-1}<1+4 \sum_{j \neq i} P_{i j}^{2}=1+4\left(P_{i i}-P_{i i}^{2}\right)<2$

as we require.

A.3. Proof of Proposition 4.7. We now establish Proposition 4.7, giving a bound on the probability that a suitably random subspace is realizable by bounding the probability that it has coherence strictly bounded above by $1 / 2$.

Proof. It suffices to show that $\left\|P_{\mathcal{U}} e_{i}\right\|_{2}^{2} \leq(1-2 \epsilon)(1 / 2-\epsilon)=1 / 2-2 \epsilon^{2}<1 / 2$ for all $i$ with high probability. The main observation we use is that if $\mathcal{U}$ is a random $r$-dimensional subspace of $\mathbb{R}^{n}$ and $x$ is any fixed vector with $\|x\|_{2}=1$, then $\left\|P_{\mathcal{U}} x\right\|_{2}^{2} \sim$ $\beta(r / 2,(n-r) / 2)$, where $\beta(p, q)$ denotes the beta distribution [13]. In the case where $r=(1 / 2-\epsilon) n$, using a tail bound for $\beta$ random variables [13] we see that if $x \in \mathbb{R}^{n}$ is fixed and $r>3 / \epsilon^{2}$, then

$$
\operatorname{Pr}\left[\left\|P_{\mathcal{U}} x\right\|_{2}^{2} \geq(1+2 \epsilon)(1 / 2-\epsilon)\right]<\frac{1}{a_{\epsilon}} \frac{1}{\left(\pi\left(1 / 4-\epsilon^{2}\right)\right)^{1 / 2}} n^{-1 / 2} e^{-a_{\epsilon} k},
$$


where $a_{\epsilon}=\epsilon-4 \epsilon^{2} / 3$. Taking a union bound over $n$ events, as long as $r>3 / \epsilon^{2}$

$$
\begin{aligned}
\operatorname{Pr}[\mu(\mathcal{U}) \geq 1 / 2] & \leq \operatorname{Pr}\left[\left\|P_{\mathcal{U}} e_{i}\right\|_{2}^{2} \geq(1-2 \epsilon)(1 / 2-\epsilon) \text { for some } i \in[n]\right] \\
& \leq n \cdot \frac{1}{a_{\epsilon}\left(\pi\left(1 / 4-\epsilon^{2}\right)\right)^{1 / 2}} n^{-1 / 2} e^{-a_{\epsilon} k}=\bar{c} n^{1 / 2} e^{-\tilde{c} n}
\end{aligned}
$$

for appropriate positive constants $\bar{c}$ and $\tilde{c}$.

Appendix B. Using complex scalars. In this appendix we briefly discuss the analogues of our main results when using complex rather than real scalars. If $z \in \mathbb{C}$ we denote by $\Re(z)$ and $\Im(z)$ its real and imaginary parts and by $|z|=\left(\Re(z)^{2}+\Im(z)^{2}\right)^{1 / 2}$ its modulus. An $n \times n$ Hermitian matrix $X$ is positive semidefinite if $v^{H} X v \geq 0$ for all $v \in \mathbb{C}^{n}$. Note that $X$ is positive semidefinite if and only if the real symmetric $2 n \times 2 n$ matrix

$$
\tilde{X}:=\left[\begin{array}{cc}
\Re(X) & \Im(X) \\
-\Im(X) & \Re(X)
\end{array}\right]
$$

is positive semidefinite. We call Hermitian positive semidefinite matrices with all diagonal elements equal to one complex correlation matrices. We focus here on establishing sufficient conditions for the complex analogue of realizability.

Definition B.1. A subspace $\mathcal{U}$ of $\mathbb{C}^{n}$ is $\mathbb{C}$-realizable if there is an $n \times n$ complex correlation matrix $Q$ such that $\mathcal{N}(Q) \supseteq \mathcal{U}$.

Note that the set of complex correlation matrices is invariant under conjugation by diagonal unitary matrices (i.e., diagonal matrices with all diagonal elements having modulus one).

B.1. Complex analogue of Theorem 3.4. As before, we say $u \in \mathbb{C}^{n}$ is $\mathbb{C}$ balanced if $\left|u_{i}\right| \leq \sum_{j \neq i}\left|u_{j}\right|$ for all $i \in[n]$. A subspace $\mathcal{U}$ of $\mathbb{C}^{n}$ is $\mathbb{C}$-balanced if all its elements are $\mathbb{C}$-balanced

Theorem B.2. If a subspace $\mathcal{U}$ of $\mathbb{C}^{n}$ is $\mathbb{C}$-realizable, then it is $\mathbb{C}$-balanced. If a subspace $\mathcal{U}$ of $\mathbb{C}^{n}$ is $\mathbb{C}$-balanced and $\operatorname{dim}(\mathcal{U})=1$, then it is $\mathbb{C}$-realizable.

Proof. Let $u \in \mathbb{C}^{n}$ span the one-dimensional subspace $\mathcal{U}$ of $\mathbb{C}^{n}$ and let $|u| \in \mathbb{R}^{n}$ be such that $|u|_{i}=\left|u_{i}\right|$. Clearly $u$ is $\mathbb{C}$-balanced if and only if $|u|$ is balanced or, equivalently, $\operatorname{span}_{\mathbb{R}}\{|u|\}$ is realizable. Now $\operatorname{span}_{\mathbb{R}}\{|u|\}$ is realizable if and only if $\operatorname{span}_{\mathbb{C}}\{|u|\}$ is $\mathbb{C}$-realizable. (To see this note that if $Q$ is a complex correlation matrix with $Q|u|=0$, then $\Re(Q)$ is a correlation matrix with $\Re(Q)|u|=\Re(Q|u|)=0$.) Finally note that $\operatorname{span}_{\mathbb{C}}\{|u|\}$ is realizable if and only if $\operatorname{span}_{\mathbb{C}}\{u\}$ is realizable. This is because there is a diagonal unitary matrix $D$ such that $D u=|u|$, so if $M$ is a complex correlation matrix with $M|u|=0$, then $Q=D M D^{H}$ is a complex correlation matrix with $Q u=0$.

The above argument also establishes that if $\mathcal{U}$ is $\mathbb{C}$-realizable, then it is $\mathbb{C}$ balanced, as every element of a $\mathbb{C}$-realizable subspace spans a one-dimensional $\mathbb{C}$ realizable subspace and so is $\mathbb{C}$-balanced.

B.2. Complex analogue of Theorem 4.2. We could establish the complex analogue of Theorem 4.2 by appropriately modifying the proof given in section 4 . We take a different approach, instead relating the $\mathbb{C}$-realizability of a subspace of $\mathbb{C}^{n}$ to the block realizability of a related subspace of $\mathbb{R}^{2 n}$.

Definition B.3. If $\mathcal{U}$ is a subspace of $\mathbb{C}^{n}$ define a subspace $\tilde{\mathcal{U}}$ of $\mathbb{R}^{2 n}$ by

$$
\tilde{\mathcal{U}}=\operatorname{span}\left\{\left[\begin{array}{c}
\Re(u) \\
-\Im(u)
\end{array}\right],\left[\begin{array}{l}
\Im(u) \\
\Re(u)
\end{array}\right]: u \in \mathcal{U}\right\} .
$$


Note that $\tilde{\mathcal{U}}$ is invariant under multiplication by $\left[\begin{array}{cc}0 & I \\ -I & 0\end{array}\right]$, corresponding to $\mathcal{U}$ being closed under multiplication by the complex unit. Observe that if $Q$ is a complex correlation matrix, then $\Im(Q)$ has zero diagonal, so $\tilde{Q}$ (defined in (B.1)) is an element of the $\mathcal{P}$-elliptope for the partition $\mathcal{P}=\{\{1, n+1\},\{2, n+2\}, \ldots,\{n, 2 n\}\}$ of $[2 n]$. Through this partition of $[2 n]$ we can relate realizability properties of $\mathcal{U}$ and $\tilde{\mathcal{U}}$.

Lemma B.4. A subspace $\mathcal{U}$ of $\mathbb{C}^{n}$ is $\mathbb{C}$-realizable if and only if $\tilde{\mathcal{U}}$ is $\mathcal{P}$-realizable.

Proof. If $Q$ is a complex correlation matrix such that $\mathcal{N}(Q) \supseteq \mathcal{U}$, then $\tilde{Q}$ (as defined in (B.1)) is in the $\mathcal{P}$-elliptope and it is straightforward to check that $\mathcal{N}(\tilde{Q}) \supseteq \tilde{\mathcal{U}}$.

On the other hand suppose there is an element $\left[\begin{array}{cc}A & B \\ B^{T} & C\end{array}\right]$ of the $\mathcal{P}$-elliptope with nullspace containing $\tilde{\mathcal{U}}$. It is straightforward to check that the Hermitian matrix $Q=(A+C) / 2+i\left(B-B^{T}\right) / 2$ has unit diagonal and satisfies $\mathcal{N}(Q) \supseteq \mathcal{U}$. It remains to show that $Q$ is Hermitian positive semidefinite. To see this note that

$$
\left[\begin{array}{cc}
A+C & B-B^{T} \\
B^{T}-B & C+A
\end{array}\right]=\left[\begin{array}{ll}
I & 0 \\
0 & I
\end{array}\right]\left[\begin{array}{cc}
A & B \\
B^{T} & C
\end{array}\right]\left[\begin{array}{cc}
I & 0 \\
0 & I
\end{array}\right]^{T}+\left[\begin{array}{cc}
0 & I \\
-I & 0
\end{array}\right]\left[\begin{array}{cc}
A & B \\
B^{T} & C
\end{array}\right]\left[\begin{array}{cc}
0 & I \\
-I & 0
\end{array}\right]^{T}
$$

which is clearly positive semidefinite whenever $\left[\begin{array}{rr}A & B \\ B^{T} & C\end{array}\right] \succeq 0$.

Define the complex coherence of a subspace $\mathcal{U}$ of $\mathbb{C}^{n}$ as $\mu_{\mathbb{C}}(\mathcal{U})=\max _{i \in[n]}\left\|P_{\mathcal{U}} e_{i}\right\|_{2}^{2}$. Note that it follows directly from the definitions that $\mu_{\mathbb{C}}(\mathcal{U})=\mu_{\mathcal{P}}(\tilde{\mathcal{U}})$. Finally, the complex version of Theorem 4.2 is as follows.

Theorem B.5. If $\mathcal{U}$ is a subspace of $\mathbb{C}^{n}$ and $\mu_{\mathbb{C}}(\mathcal{U})<1 / 2$, then $\mathcal{U}$ is $\mathbb{C}$-realizable.

Proof. Since $\mu_{\mathbb{C}}(\mathcal{U})=\mu_{\mathcal{P}}(\tilde{\mathcal{U}})$ we have that

$$
\mu_{\mathbb{C}}(\mathcal{U})<1 / 2 \Longrightarrow \mu_{\mathcal{P}}(\tilde{\mathcal{U}})<1 / 2 \Longrightarrow \tilde{\mathcal{U}} \text { is } \mathcal{P} \text {-realizable } \Longrightarrow \mathcal{U} \text { is } \mathbb{C} \text {-realizable }
$$

where the last two implications follow from Corollary 5.11 and Lemma B.4.

Acknowledgments. The authors would like to thank Prof. Sanjoy Mitter for helpful discussions and the anonymous reviewers for carefully reading the manuscript and providing many helpful suggestions.

\section{REFERENCES}

[1] A.A. Albert, The matrices of factor analysis, Proc. Natl. Acad. Sci. USA, 30 (1944), pp. 9095.

[2] J. BriËt, F. De Oliveira Filho, and F. Vallentin, Grothendieck Inequalities for Semidefinite Programs with Rank Constraint, preprint, arXiv:1011.1754, 2010.

[3] A.E. Brouwer and J. Draisma, Equivariant Gröbner bases and the Gaussian two-factor model, Math. Comp., 80 (2011), pp. 1123-1133.

[4] E.J. CANDÈs, X. Li, Y. MA, AND J. Wright, Robust principal component analysis?, J. ACM, 58 (2011), pp. 11:1-11:37.

[5] E.J. CANDÈs AND B. ReCht, Exact matrix completion via convex optimization, Found. Comput. Math., 9 (2009), pp. 717-772.

[6] V. Chandrasekaran, S. Sanghavi, P.A. Parrilo, and A.S. Willsky, Rank-sparsity incoherence for matrix decomposition, SIAM J. Optim., 21 (2011), pp. 572-596.

[7] G. Della Riccia And A. Shapiro, Minimum rank and minimum trace of covariance matrices, Psychometrika, 47 (1982), pp. 443-448.

[8] C. Delorme and S. PoljaK, Combinatorial properties and the complexity of a max-cut approximation, European J. Combin., 14 (1993), pp. 313-333.

[9] A.P. Dempster, N.M. Laird, And D.B. Rubin, Maximum likelihood from incomplete data via the EM algorithm, J. R. Stat. Soc. Ser. B Stat. Methodol., 39 (1977), pp. 1-38.

[10] D.L. Donoho And X. Huo, Uncertainty principles and ideal atomic decomposition, IEEE Trans. Inform Theory, 47 (2001), pp. 2845-2862. 
[11] M. Drton, B. Sturmfels, and S. Sullivant, Algebraic factor analysis: Tetrads, pentads and beyond, Probab. Theory Related Fields, 138 (2007), pp. 463-493.

[12] M. Fazel and J. Goodman, Approximations for Partially Coherent Optical Imaging Systems, Technical report, Stanford University, Stanford, CA, 1998.

[13] P. Frankl and H. Maehara, Some geometric applications of the beta distribution, Ann. Inst. Statist. Math., 42 (1990), pp. 463-474.

[14] M.X. Goemans And D.P. Williamson, Improved approximation algorithms for maximum cut and satisfiability problems using semidefinite programming, J. ACM, 42 (1995), pp. 11151145.

[15] R. Grone, S. Pierce, And W. Watkins, Extremal correlation matrices, Linear Algebra Appl., 134 (1990), pp. 63-70.

[16] N.J. Higham, Computing the nearest correlation matrix-a problem from finance, IMA J. Numer. Anal., 22 (2002), pp. 329-343.

[17] R.E. Kalman, Identification of noisy systems, Russian Math. Surveys, 40 (1985), pp. 25-42.

[18] H. Krim And M. Viberg, Two decades of array signal processing research, IEEE Signal Process. Mag., 13 (1996), pp. 67-94.

[19] M. Laurent And S. POLJAK, On a positive semidefinite relaxation of the cut polytope, Linear Algebra Appl., 223 (1995), pp. 439-461.

[20] M. Laurent and S. Poljak, On the facial structure of the set of correlation matrices, SIAM J. Matrix Anal. Appl., 17 (1995), pp. 530-547.

[21] W. Ledermann, On a problem concerning matrices with variable diagonal elements, Proc. Roy. Soc. Edinburgh, 60 (1940), pp. 1-17.

[22] M. Mesbahi and G.P. Papavassilopoulos, On the rank minimization problem over a positive semidefinite linear matrix inequality, IEEE Trans. Automat. Control, 42 (1997), pp. 239243.

[23] B. Recht, M. FAzel, And P.A. PARRIlo, Guaranteed minimum-rank solutions of linear matrix equations via nuclear norm minimization, SIAM Rev., 52 (2010), pp. 471-501.

[24] J. Saunderson, Subspace Identification via Convex Optimization, Master's thesis, Massachusetts Institute of Technology, Cambridge, MA, 2011.

[25] P. Shah and V. Chandrasekaran, Group Symmetry and Covariance Regularization, preprint, arXiv:1111.7061, 2011.

[26] A. ShapIRo, Rank-reducibility of a symmetric matrix and sampling theory of minimum trace factor analysis, Psychometrika, 47 (1982), pp. 187-199.

[27] A. Shapiro, Weighted minimum trace factor analysis, Psychometrika, 47 (1982), pp. 243-264.

[28] A. SHAPIRo, Identifiability of factor analysis: Some results and open problems, Linear Algebra Appl., 70 (1985), pp. 1-7.

[29] C. Spearman, "General intelligence," objectively determined and measured, American J. Psychol., (1904), pp. 201-292.

[30] L. VAndenBerghe ANd S. Boyd, Semidefinite programming, SIAM Rev., 38 (1996), pp. 49-95.

[31] J.A. WALters, Nonnegative matrix equations having positive solutions, Math. Comp., (1969), p. 827.

Copyright (c) by SIAM. Unauthorized reproduction of this article is prohibited. 